\title{
Unknown negative genes in the positive RNA genomes of coronaviruses
}

Prof. Zhirnov Oleg ${ }^{1}$, O P Zhirnov ${ }^{2,3}$, and S V Poyarkov ${ }^{3}$

${ }^{1}$ Affiliation not available

${ }^{2}$ The D.I.Ivanovsky Institute of Virology, The N.F. Gamaleya National Research Center of Epidemiology and Microbiology

${ }^{3}$ The Russian-German Academy of medico-social and biotechnological sciences

November 24, 2020

\begin{abstract}
Coronavirus family has a single-stranded RNA genome encoding 25-30 proteins in different viruses by the mechanism of positivesense strategy. Extended open reading translation frames (genes) were found to locate under a negative-sense polarity in all coronaviruses genomes. These negative-sense genes varies in the range of 150-450 nt to encode negative genes polypeptides (NGP) with mol. wt. 5-30 kDa. It implies that coronaviruses besides positive genome strategy may have "a dark side of the Moon" expressing genes and virions through the negative strategy. It is noteworthy, that positive- and negative-sense genes colocolized in the same RNA regions of coronavirus genome, so called stacking genes. Ambisense stacking of genes in coronavirus genomes significantly increases virus diversity, genetic potential and extend virus-host adaptation pathway possibilities.
\end{abstract}

\section{Hosted file}

Zhirnov text 23-11-2020 +Figures.pdf available at https://authorea.com/users/376789/articles/ 493682-unknown-negative-genes-in-the-positive-rna-genomes-of-coronaviruses 